\title{
Finite strain compressive behaviour of CNT/epoxy nanocomposites: 2D versus 3D RVE-based modelling
}

\author{
David Weidt ${ }^{1,2,3}$, Łukasz Figiel ${ }^{4,5, a}$ \\ ${ }^{1}$ Department of Mechanical, Aeronautical and Biomedical Engineering, University of Limerick, \\ Ireland \\ ${ }^{2}$ Department of Civil Engineering and Materials Science, University of Limerick, Ireland \\ ${ }^{3}$ Materials \& Surface Science Institute, University of Limerick, Ireland \\ ${ }^{4}$ Centre of Molecular and Macromolecular Studies, Polish Academy of Sciences, Sienkiewicza 112, \\ 90-363 Lodz, Poland \\ ${ }^{5}$ School of Engineering, University of Portsmouth, PO1 3DJ, United Kingdom
}

Keywords: CNT/epoxy nanocomposites, Compression, Finite strains, Strain-rate dependence, Finite element modelling, Representative volume element

\begin{abstract}
The macroscopic finite strain compressive behaviour of CNT/epoxy nanocomposites at quasi-static and high strain-rates was predicted and compared using 2D and 3D RVE approaches, a nonlinear and rate-dependent model for the epoxy matrix and nonlinear numerical homogenisation. Effects of CNT orientation, CNT volume fraction, CNT aspect ratio on the nanocomposite response were predicted and analysed, to compare 2D and 3D modelling approaches and obtain an insight into the nonlinear finite compressive behaviour of CNT/epoxy systems. It was found that the nanocomposite nonlinear compressive stress-strain response cannot be accurately captured by 2D RVEs (assuming the plane strain condition), when compared to 3D RVEs, primarily because of the stress transfer effect and the interparticle interaction accurately captured only in 3D. In general, 3D models predicted that the increasing CNT aspect ratio, CNT volume fraction and CNT alignment enhance the nonlinear finite strain compressive response by increasing the yield peak true stress and changing the post-yield deformation behaviour from softening to hardening. Also, the CNT alignment was identified as the major factor for enhancing the nonlinear stress-strain response at both quasi-static and impact rates of strain.
\end{abstract}

\section{Introduction}

Epoxy resin-based carbon fibre-reinforced polymer (CFRP) laminates are widely used in lightweight structures in the aerospace and wind-turbine industries. They became very attractive due to their good specific stiffness/strength, improved fatigue life and corrosion resistance, when compared to metals. All these unique material characteristics promise to meet current demands for the reduction of fuel consumption, efficient energy generation and the long-term structural and environmental durability. However, one of the remaining issues, which still compromise the use of epoxy-based laminates in high-performance applications, is their relatively brittle behaviour when exposed to in-service impact events such as those caused by foreign objects. Therefore, improvement of the energy absorption characteristics of those materials is an important research goal for material scientists and engineers working with composite materials.

Over the last two decades, attention in composites research has been shifted to the use of nanofillers, such as carbon nanotubes (CNTs), to improve the toughness of epoxy matrices and their 
composites. The exceptional mechanical properties of CNTs (e.g. stiffness, resilience) $[1,2]$ and their specific surface area which is several orders of magnitude larger than conventional microfillers [3] offer means to enhance the mechanical performance of epoxy at low CNT concentrations. This also includes the enhancement of energy absorption characteristics, which are brought by two main deformation mechanisms: (1) enhanced nonlinear finite deformation of epoxy in compression due to the presence of CNTs [4] and (2) CNT crack bridging mechanisms [5,6] accompanied by CNT pullout and interface debonding in tension. This work addresses the first of those two deformation mechanisms.

In contrast to the brittle behaviour of epoxy in tension, a prominent feature of epoxy undergoing compressive loading is its ductility [7]. Three distinct regimes can be recognised within the stressstrain curve. The linear elastic regime and yield is followed by a strain softening regime and a subsequent strain hardening regime. The nonlinear deformation of CNT/epoxy in compression depends on the manufacturing method-induced nanocomposite morphology (e.g. CNT distribution and CNT orientation), material composition (e.g. CNT volume fraction) and the deformation rate applied. Theoretically, CNTs aligned and optimally distributed within the epoxy matrix promise to provide the maximum enhancement in mechanical properties in the direction of CNT alignment. However, obtaining homogenous CNT dispersion and CNT alignment within polymeric matrices remains a significant research challenge. Despite some successful attempts of controlling the CNT alignment $[8,9,10,11]$, only few studies investigated the effect of aligned CNTs in epoxy on the nonlinear compressive response (see for example [12]). Likewise, the nonlinear compressive response of $\mathrm{CNT} /$ polymer composites at high strain rates has only been sparsely researched $[13,14,15]$.

Advanced material modelling can complement experimental work, and assist experimentalists in exploring systematically the relationship between the process-induced nanocomposite morphology and rate-dependent nonlinear behaviour of CNT-based nanocomposites. Despite its practical importance, only a few numerical studies have considered the nonlinear compressive response of CNT/epoxy or CNT/polymer composites. Nanocomposite behaviour (e.g. CNT instability and damping behaviour) under compressive loading was predicted using molecular dynamics (MD) and multiscale finite element (FE) modelling $[16,17,18]$. The proposed models did not however consider sufficiently large deformations (important as an energy absorbing mechanism), strain-rate dependence (occurring during impact), and nanocomposite morphological effects (e.g. CNT orientation) for the prediction of the nanocomposite mechanical response.

Therefore, this work seeks to determine the influence of material composition, nanocomposite morphology, and strain rate on the nonlinear compressive response of CNT/epoxy nanocomposites using the representative volume element (RVE) concept and nonlinear FE-based modelling. The RVE concept was followed in this work to account explicitly for the nanocomposite material composition and morphology, and study the influence of CNT volume fraction, CNT aspect ratio and CNT orientation on the nanocomposite response. A nonlinear finite strain constitutive model, developed and validated previously for the epoxy resin [19], was integrated with RVEs and implemented into the FE framework (ABAQUS) to capture the rate-dependent behaviour of the nanocomposite matrix ranging from quasi-static to impact strain rates. Two- and three-dimensional (2D and 3D) RVE-based computational models were developed to study their accuracy and computational-efficiency in capturing the nonlinear, finite strain response of the nanocomposites. This paper is structured as follows. Modelling methodology and its numerical implementation are introduced first. Then, results of a mesh convergence analysis and RVE size study are shown. Subsequently, 2D and 3D RVE-based predictions of the finite strain behaviour of CNT/epoxy nanocomposites are presented and discussed with respect to nanocomposite morphological characteristics, strain rate, computational effort and experimental results. 


\section{Framework of modelling}

The applied modelling approach (see Fig. 1) involves four length scales of the nanocomposite: (1) the nanoscale, where CNT properties were derived using molecular mechanics; (2) the microscale, where the discrete CNT and discrete epoxy were represented by a homogenised solid cylinder and continuous medium, respectively; (3) the mesoscale, where the morphology of the nanocomposite was reconstructed using RVEs under assumptions of global periodicity; and (4) the macroscale at which the nanocomposite response (stress-strain curves) was predicted. These stress-strain curves might serve as further input in a bottom-up multiscale approach for the prediction of the impact performance of CNT/epoxy-based composite systems. The parameters captured by each scale are presented in Table 1.

Continuum theory was assumed to hold down to the scale at which the nanocomposite morphology can be captured with an RVE. This offers a compromise between accuracy and computational time. The RVE, as a building block of the macroscopic nanocomposite, allows for the prediction of the macroscopic behaviour using a numerical nonlinear homogenization technique.

\subsection{Representative Volume Elements (RVEs)}

An RVE is defined here as the minimum size of a heterogeneous material model that ensures a sufficiently accurate representation of the macroscopic material behaviour. Hence, it has to contain a minimum number of CNTs and deformation mechanisms to represent statistically the response of the macroscopic nanocomposite.

The morphology of CNT/epoxy nanocomposites is quite complex and its reconstruction using RVEs poses significant challenges even when CNTs are simplified as straight and non-bundled short fibres of different distributions and orientations, as is the case in this work. 3D RVEs are a natural choice to capture such morphologies, but they require a large number of finite elements and computational resources. On the other hand, 2D RVEs are computationally more efficient than 3D RVEs, but they can compromise the accuracy of their predictions.

Therefore, two types of RVEs were considered and compared here: (a) 2D RVEs assuming the plane strain condition (see Fig. 2a and 2b) and (b) 3D RVEs (see Fig. 2c and 2d). 3D RVEs were chosen because of their ability to accurately capture the CNT geometry with two 'nanometre' dimensions in the plane of the circular cross-section and one dimension in 'micrometre' scale in CNT axis direction. As a consequence, 3D RVEs exhibit the correct ratio of number of CNTs to RVE volume for a given CNT volume fraction. On the other hand, 2D RVEs were chosen for their computational efficiency on the account of the following assumptions: (a) the third dimension of 2D RVEs under plane strain is neglected (set to unity here), and only one 'nanoscale' dimension is captured (i.e. thickness of a CNT) - hence, the CNT shape is captured accurately in the x-y plane only, and the ratio of the number of CNTs to 2D RVE volume for a given CNT volume fraction depends here on the scaling of the CNT dimensions, and (b) the well-known constraint imposed on the out-of-plane strains (in the z-direction here), which is expected to provide a stiffer macroscopic response.

Both, 2D and 3D RVEs assumed two different CNT orientations: (1) aligned (in the x-direction) (see Fig. 2a and 2c), and (2) misaligned (random) (see Fig. 2b and 2d). The sides of each of the RVEs used possess equal lengths, except for 3D RVEs of randomly distributed and aligned CNTs (see Fig. 2c), where a cuboidal cell was chosen over a cube in order to save computational costs related to the required number of CNTs. For example, a cubic RVE with a minimum edge length of two times the CNT length, a CNT volume fraction of 0.01 and an aspect ratio of 50, would require 255 CNTs. In contrast, cuboidal RVEs would need to be populated with a significantly smaller number of CNTs depending on the ratio of the longer to shorter RVE edge. The ratio of CNT dimensions to RVE dimension is much smaller in the transverse direction. Hence, the long side of the cuboidal RVE was assumed to be crucial for its size. The length of the short edges of the 
cuboidal RVE were set equal to the short edges in an RVE of 20 CNTs that exhibits a long edge length of $2.5 \times \mathrm{CNT}$ length.

In-house developed scripts were used to build 2D and 3D RVEs of the nanocomposite. One of the key-functions included in these codes was the random positioning and the random alignment of CNTs in a squared, cubic or cuboidal geometric cell representing the RVE. A typical acceptancerejection algorithm was employed to populate RVEs with cylinder-like objects representing homogenised CNTs (see Section 2.1.2). For this, checks for intersections with previously generated objects were carried out either resulting in the deletion or inclusion of the newly generated object. This procedure continued until the requested number of CNTs was incorporated. The assumption of morphological periodicity imposed additional conditions on the objects intersecting the boundaries of the RVE - those objects were divided into inner and outer parts, and the latter ones were translated to the inside of the opposite RVE edges. A review on numerical generation of random fibre composites may be found in [21].

A non-contacting distribution of CNTs was enforced by assuming a minimum distance between CNT surfaces, which was set equal to the CNT diameter. Accounting for overlapping and connection of CNTs may affect the stiffness of the nanocomposite as shown by Makvandi and Öchsner [22]. Additionally, the effect of CNT agglomerations is not considered, although the level of CNT agglomeration was found to have a significant effect on the nonlinear stress-strain response of CNT nanocomposites [23]. However, CNT agglomerations may be minimized by using an appropriate dispersion method [5], surface functionalisation [24], low CNT volume fractions [25] and low CNT aspect ratios [25,26].

The meshing of the RVEs was performed using the ABAQUS built-in free meshing algorithm. Periodic boundary conditions require that relevant nodal coordinates of finite elements lying on opposite faces (or edges) of an RVE are the same before and during deformation. In the case of 3D RVEs, the matrix material was discretised by 10-node quadratic tetrahedron elements (C3D10) and the CNT by 20-node quadratic brick elements (C3D20). 6-node quadratic plane strain triangle elements (CPE6) were used in 2D RVEs for both, CNT and matrix discretisation.

\subsubsection{Carbon nanotube representation in RVEs}

The concept of the effective (homogenised) representations of single-walled CNTs (SWCNTs) [27] was followed by modelling SWCNTs as homogenised solid fibres of the given aspect ratio. This SWCNT representation is computationally more efficient when compared with a discrete, tubular representation of a SWCNT. In general, the effective CNT is transversely isotropic with five independent elastic constants. In the present work, it was assumed for simplicity that the material behaviour of each homogenised CNT is linear elastic and isotropic, i.e. it can be described by only two elastic constants: Young's modulus $E^{\text {eff }}$ and Poisson's ratio $v$, which were determined from the following equations for zig-zag SWCNTs based on harmonic potentials [27]:

$$
\begin{gathered}
E^{\mathrm{eff}}=\frac{4 \sqrt{3} C_{\rho}}{(R / 2)\left[9+3\left(C_{\rho} r_{0}^{2} / C_{\theta}\right) /(2 \eta)\right]}, \\
v=\frac{-1+\left(C_{\rho} r_{0}^{2} / C_{\theta}\right) /(2 \eta)}{3+\left(C_{\rho} r_{0}^{2} / C_{\theta}\right) /(2 \eta)}, \\
\eta=\frac{14+12 \cos (\pi / n)-2 \cos ^{2}(\pi / n)}{10+4 \cos (\pi / n)-6 \cos ^{2}(\pi / n)}
\end{gathered}
$$

where $C_{\rho}=742 \mathrm{nN} / \mathrm{nm}$ [27] is the bond stretching force constant, $C_{\theta}=1.42 \mathrm{nNnm}$ [27] is the bond angle variation constant, $r_{0}=0.1421 \mathrm{~nm}$ is the C-C bond length, $R \approx 5 \mathrm{~nm}$ is the CNT radius and $n=128$ is the corresponding chirality of a zig-zag SWCNT. Effective Young's modulus and Poisson's ratio were calculated to be equal to $143.62 \mathrm{GPa}$ and 0.16 , respectively. 


\subsubsection{Constitutive model for the epoxy matrix in the RVE}

The physically-based constitutive 'glass-rubber' model proposed and experimentally-validated for thermosetting resins [19] was chosen to represent the nonlinear strain rate-dependent behaviour of the epoxy matrix. The model assumes two sources of energy arising from perturbation of interatomic potentials (relaxed by thermally activated flow events) and from entropy-elastic perturbation of molecular conformations (arising from preferred molecular alignment) [19]. As a result, there are two deviatoric stress components: (1) bond-stretching stress and (2) conformational stress (described in terms of isotropic hyperelasticity). The model is able to capture strain-rate dependence of the yield peak, strain softening, and to account for the adiabatic heating (active upon impact rates of deformation). The constitutive model incorporates those features by applying the Eyring and fictive temperature concepts, and a representation of the plastic-strain-induced rejuvenation [19].

The model was described within the framework of nonlinear continuum, and hence its kinematics is driven by the deformation gradient tensor, while its rate-dependency is driven by the viscous part of the deformation rate tensor. The model was implemented into the nonlinear FE framework using a user subroutine UMAT (ABAQUS/Standard). Model parameters obtained for the standard bisphenol A resin were used in these simulations [19].

\subsubsection{CNT/epoxy interface/interphase in the RVE}

The CNT and epoxy were assumed to be perfectly bonded in this work. However, this is only an approximation to the real picture of the interface behaviour in CNT/epoxy nanocomposites, where the strength of interactions between CNT and epoxy varies depending on the CNT surface functionalisation [28]. Also, it was assumed that the epoxy properties in the vicinity of CNTs are the same as in the bulk. This is again an approximation as the presence of CNTs can change the epoxy dynamics and/or disrupt its network near the CNT [29]. Incorporation of an interface/interphase can significantly change the stress transfer efficiency $[30,31,32]$ and affect the nonlinear behaviour of the nanocomposite $[23,33]$.

\subsection{Computational homogenisation: RVE-to-macro transition}

Computational homogenisation was applied to calculate macroscopic stresses from each RVE. The nanocomposite was assumed to be globally periodic, i.e. consisting of the same repeatable RVE. The macroscopic deformation was considered as strain-controlled and it was assumed that the applied macroscopic strain $\boldsymbol{\varepsilon}_{\mathrm{M}}$ is uniform such that each RVE experienced the same strain. The strain within each RVE boundary consisted of the linear contribution resulting from the macroscopic strain, and the periodic component, which arises from the nanocomposite morphology. Therefore, the resulting displacements in the RVE are given as:

$$
\boldsymbol{u}_{\mathrm{RVE}}=\boldsymbol{u}_{\mathrm{M}}+\boldsymbol{u}_{\mathrm{per}},
$$

where the displacement due to the macroscopic strain $\boldsymbol{\varepsilon}_{\mathrm{M}}$ is given through the well-known linear relation:

$$
\boldsymbol{u}_{\mathrm{M}}=\boldsymbol{\varepsilon}_{\mathrm{M}} \boldsymbol{x}_{\mathrm{RVE}}
$$

where $\boldsymbol{x}_{\mathrm{RVE}}$ denotes the relevant position within the RVE, and the periodic boundary conditions were applied to link the opposite boundaries (faces and/or edges) of the RVE to ensure they undergo the same displacements according to:

$$
\boldsymbol{u}_{\mathrm{RVE}}^{+}-\boldsymbol{u}_{\mathrm{RVE}}^{-}=\boldsymbol{u}_{\mathrm{M}}^{+}-\boldsymbol{u}_{\mathrm{M}}^{-}=\varepsilon_{\mathrm{M}}\left(\boldsymbol{x}_{\mathrm{RVE}}^{+}-\boldsymbol{x}_{\mathrm{RVE}}^{-}\right),
$$

where symbols ' + ' and '-' represent corresponding points on two opposite faces and/or edges of the RVE boundary.

In this work, the RVE domain was separated into corner nodes $\left(I_{C}=1-8\right)$, faces (Top, Bottom, Left, Right, Front, Rear) and edges. The corner node 1 was constrained in all directions to prevent rigid 
body movement and the Eqs. (5)-(6) were applied to the relevant RVE regions to simulate the macroscopic uniaxial compression of the nanocomposite in the x-direction. Fig. 3 and Table 2 show the displacements and constraints applied to 2D and 3D RVEs.

The macroscopic Cauchy stresses $\sigma_{\mathrm{M}}$ were calculated from the forces resulting from the surface tractions caused by the displacements imposed on the RVE boundaries (see Eq. (4)):

$$
\boldsymbol{\sigma}_{M}=\frac{1}{V_{\mathrm{RVE}}} \int_{V_{\mathrm{VVE}}} \boldsymbol{\sigma}_{\mathrm{RVE}}(x) d V=\frac{1}{V_{\mathrm{RVE}}} \int_{\text {rRVE }} \boldsymbol{t}_{\mathrm{RVE}} \otimes \boldsymbol{x}_{\mathrm{RVE}} d \Gamma,
$$

For the boundary conditions applied in this work (see Fig. 3 and Table 2), Eq. (7) results in the macroscopic stress component $\sigma_{\mathrm{xx}}=V_{\mathrm{RVE}}^{-1} f_{\mathrm{x}(2)} x_{\mathrm{x}(2)}$ where $f_{\mathrm{x}(2)}$ and $x_{\mathrm{x}(2)}$ denote force and position of the node 2 (see Fig. 3).

\section{Results and discussion}

The developed 2D and 3D computational models were used to predict the large strain compressive response of CNT/epoxy for different material compositions and nanocomposite morphologies. The results are presented and discussed in this section. First a mesh convergence study is addressed followed by the determination of the RVE size and ensemble size. Subsequently, the results section is focussed on the investigation of the 2D and 3D modelling approach with respect to the effects of CNT aspect ratio, CNT volume fraction and strain-rate effects on the large strain nanocomposite response. For this purpose, stress-strain parameters introduced in Section 3.2 were analysed. Furthermore the computational effort of 2D and 3D RVEs is presented and finally the predictions are compared against experimental data from literature.

\subsection{Mesh convergence}

The mesh density can influence FE results significantly, especially when nonlinear FE simulations are performed. Thus, the sensitivity of the stress-strain behaviour to the density of the FE mesh was studied for 2D and 3D models (CNT aspect ratio of 50, CNT volume fraction of 1\%). In order to minimise the computational time, relatively small models incorporating only five CNTs were used in the mesh study. 2D models allowed for constant and fine mesh densities due to their computational efficiency (relatively small number of degrees of freedom and element integration points). Therefore, the mesh was refined globally by reducing the average element size. For the sake of computational cost reduction, 3D models were only refined in regions of interest, i.e. the mesh density of the matrix domain close to the CNTs was enhanced by using more elements along the CNT circumference and also along the CNT axis.

These mesh refinement strategies are denoted here by: (A) one number in $2 \mathrm{D}$, e.g. 11, which stands for the element number along the 2D CNT, and (B) a set of two numbers, e.g. 17;4 - the first number refers to the element number along the $3 \mathrm{D} \mathrm{CNT}$ axis and the second number specifies the element number along the circumference of the 3D CNT.

Additionally, and for completeness, two special cases of mesh refinement were also considered for the 3D model. First, the effect of the size growth parameter for ABAQUS built-in free meshing algorithm on the result was investigated. Apart from the element seeding at the geometric edges (e.g. RVE edges), the user can adjust a size growth parameter for interior elements (sizeGrowth), which affects the element edge lengths inside the geometric volume. The parameter was decreased from 'sizeGrowth=MAXIMUM' to 'sizeGrowth=MODERATE' (case 17;4*). Secondly, the element edge length at the model cube edges was treated as an additional factor and it was changed from $0.108 \times \mathrm{CNT}$ length to $0.077 \times \mathrm{CNT}$ length (case $17 ; 4^{* *}$ ).

The mesh density effect on 2D stress-strain curves are not shown here as the true stress-true strain curves of the two mesh extremes, i.e. mesh densities with 50 and 11 elements along the CNT axis, were coincident. Hence, Fig. 4 shows only the results of mesh study for 3D models. Moreover, as it 
was found that the linear elastic regime and yield peak stress are relatively unaffected by the tested mesh densities in the 3D model, the analysis is focused on the nonlinear portion of the 3D stressstrain behaviour.

In particular, the post-yield behaviour showed a mesh dependence. The number of elements along the CNT axis (see Fig. 4a) and along the CNT circumference (see Fig. 4b) influences the post-yield behaviour significantly. The effect of size growth parameter and the element edge length at the model cube edges is negligible. The stress-strain curves converge for the case 50;4 as further mesh refinements with more elements along the CNT axis (case 100;4) or along the CNT circumference (case 50;8) brought negligible effects. As a summary, Fig. 5 shows the convergence of the overall nanocomposite stress value (for $2 \mathrm{D}$ and $3 \mathrm{D}$ models at $15 \%$ of the overall true strain) when plotted as a function of element number.

It is noteworthy to mention that premature termination of $\mathrm{FE}$ analyses becomes a serious problem when relatively fine meshes are used, as for example obtained for the mesh refinement cases 50;4, $100 ; 4$ and 50;8. This is caused by the excessive magnitude of local stresses in the matrix (in the vicinity of CNTs) resulting from the enhanced nonlinear hyperelastic response of the constitutive model, which captures the strain hardening behaviour of the epoxy matrix. This resulted in local stresses rising rapidly with a small increase of strains, leading to convergence problems in the numerical solution. The numerical convergence may be improved by the development of a more robust constitutive tangent. However, as those local stresses exceeded the strength of pure epoxy matrix under macroscopic compression [7], continuation of the analysis would lead to unphysical results. Instead, introduction of a damage variable into the constitutive model for the epoxy matrix will be explored in the future. Therefore, case 17;4 for 3D models was finally chosen as a compromise between accuracy and achievable finite strains, which are nearly twice as much as for the case $50 ; 4$.

\subsection{RVE size and ensemble size}

RVE size and the ensemble size (number of RVE realisations per case) are important for accurate predictions of the nanocomposite response. Deterministic size effects specify the minimum volume of the RVE, provided that they converge with increasing RVE size, while statistical size effects specify the number of RVE realisations according to the required accuracy. Thus, studies with 2D and 3D RVEs composed of different numbers of CNTs were carried out to investigate deterministic and statistical RVE size effects.

Specific parameters representing subsequent stages of the stress-strain behaviour of CNT/epoxy nanocomposites were selected for the analysis of the RVE size and ensemble size: (a) the Young's modulus $E$ was defined here as initial slope of the stress-strain response of both, 2D and 3D models; (b) the yield peak stress $\sigma_{\mathrm{Y}}$ (start of material softening) or, in absence of a pronounced yield peak, the yield strength $\sigma_{\mathrm{p} 1}$ with an offset of $1 \%$ of plastic strain; (c) the minimum tangent modulus $T_{\min }$ of the whole curve; (d) the softening strain limit $\varepsilon_{\mathrm{S}}$ (the true strain value representing the end of the softening regime and the start of the hardening regime, corresponding to a stress minimum between softening and hardening); (e) the initial hardening modulus $H$ (only for 2D RVEs) defined here as secant modulus between the point (in the hardening regime), where the tangent modulus is equal to $1.5 \times 10^{8} \mathrm{~Pa}$, and the point on the graph determined by adding 0.05 to the true strain value of the first point.

All RVE-size studies were carried out for the CNT volume fraction of 1\%, which represents the highest volume fraction considered in this work and thus accounts for the maximum CNT-CNT interactions. The influence of the CNT aspect ratio on the RVE size was studied for 2D (aspect ratios of 25, 50 and 100) and for 3D (aspect ratios of 25 and 50) models. Two nanocomposite morphologies were considered: (1) 2D and 3D, with randomly oriented and randomly distributed CNTs, and (2) 3D with aligned and randomly distributed CNTs. RVE-size studies were performed for the quasi-static strain rate of $1 \times 10^{-3} \mathrm{~s}^{-1}$, as it was assumed that the conclusions will also hold for higher strain rates. 
The volume element (VE) size was varied indirectly by changing the number of incorporated CNTs. Thus, the objective was to determine a minimum number of CNTs, and to choose a number of RVE realisations (RVE ensemble size) that ensures an acceptable width of the confidence interval. All stress-strain parameters mentioned above were investigated and the number of realisations varied between 10 (for small VEs) and 3 (for large VEs).

As an example, Fig. 6a and $6 \mathrm{~b}$ shows the evolution of $E$ and $T_{\min }$ as a function of CNT number, i.e. VE size. The standard deviations of the investigated parameters of VE ensembles are represented by the error-bars. Deterministic size effects refer to variation of the means across VE sizes, while statistical size effects refer to variation of the standard deviations across VE sizes. Thus, potential deterministic size effects are indicated by effects of CNT number on the investigated parameters.

Due to the limited number of VE realisations, parametric Welch's tests (with and without rank transformation) [34] were performed to conclude upon the statistical significance of potential deterministic size effects. Unequal variances were assumed due to statistical size effects that cause the standard deviations to decrease with increasing VE size. The null hypotheses (H0) on the equality of the means of two VE sizes were tested at two significance levels of $\alpha=0.05$ (significant) and $\alpha=0.01$ (highly significant). The results of the Welch's tests are shown in Table 3. Fig. $6 \mathrm{c}$ shows how a deterministic size effect shows up in a larger slope in the post-yield regime for 3D VEs with aligned CNTs (CNT aspect ratio of 50), and previously indicated as the effect on $T_{\min }$ (see Table 3).

Investigation of the strain-hardening behaviour of the nanocomposites was only possible for 2D VEs of CNT aspect ratio of 25 and 50 because of the premature termination of simulations at early stages of the hardening response (see comment in Section 3.1).

The results suggest that the minimum number of randomly oriented CNTs of aspect ratio of 50 in 2D RVEs is 60 CNTs or higher due to a deterministic size effect affecting the hardening modulus $H$. A convergence study of the deterministic size effect on $H$ was not carried out due to computational constraints. However, in the context of comparison of 2D and 3D RVE approaches, an investigation of the hardening modulus was omitted due to premature termination of simulations of 3D RVEs. Hence, the selection of a smaller RVE size was deemed to be reasonable. The minimum number of randomly oriented CNTs with an aspect ratio of 100 in 2D RVEs is 20 CNTs. For 3D cuboidal RVEs (with aligned CNTs) a minimum number of 12 CNTs is suggested.

The confidence interval widths $(C I W)$ were calculated for the stress-strain curve parameters for various ensemble sizes (i.e. the number of VE realisations). Ensemble standard deviations were assumed to be equal for various ensemble sizes. Student's t-distribution was used due to small ensemble sizes. The results for two stress-strain curve parameters are shown in Fig. 7. For 3D RVEs composed of randomly oriented CNTs a significant reduction of the $C I W$ of the parameter $\varepsilon_{\mathrm{S}}$ was obtained by increasing the CNT number from 15 to 25 CNTs. An RVE populated with 25 CNTs possesses a normalised $C I W$ of the parameter $\sigma_{\mathrm{Y}}$ of $5 \%$ for an ensemble size of 5 . Minor improvements were obtained for this parameter by increasing the ensemble size. Hence, an RVE containing 25 CNTs of an acceptable ensemble size of 5 was chosen in the 3D case. In a similar manner, RVEs populated with $20 \mathrm{CNTs}$ and subject to 5 realisations were chosen for the 2D random orientation case, while RVEs containing 20 CNTs with 3 realisations were selected for the $3 \mathrm{D}$ aligned case.

\subsection{D versus 3D RVE-based analyses of finite strain compressive behaviour of the nanocomposite}

Finite strain compressive behaviour was predicted using the computational model (see Section 2) to elucidate effects of CNT aspect ratio, CNT volume fraction, and strain rate on stress-strain parameters (as introduced in Section 3.2). Parameter results for the single constituents are presented in Table 4. It is important to point out that the Young's modulus was defined here as initial tangent modulus, which defines the slope of the linear portion of the initial stress-strain behaviour in 2D (plane strain) and 3D. Different assumptions invoked by the 2D (plane strain) and 3D models 
1 resulted in the deviation between the initial tangent modulus $E$ in $3 \mathrm{D}$ and its counterpart $E_{\mathrm{pe}}$ in $2 \mathrm{D}$

(see Table 4), which can be described through the relationship $E=\left(1-v^{2}\right) E_{\mathrm{pe}}$ [35], where $v$ denotes Poisson's ratio. Throughout this paper we use the term Young's modulus to define the initial tangent modulus of the 2D (plane strain) and 3D stress-strain curves.

Previously selected RVE sizes (in terms of CNT numbers) and RVE ensemble sizes for randomly oriented CNTs (see Section 3.2) were used for RVEs populated with aligned CNTs. For 2D RVEs, the RVE size for randomly oriented and aligned CNTs was assumed to be the same.

\subsubsection{Effect of CNT aspect ratio}

The effect of CNT aspect ratio is shown in Figs. 8 and 9. Both figures show results obtained for RVEs containing randomly distributed and randomly oriented CNTs volume fraction of $1 \%$ and subjected to quasi-static strain rates $\left(1 \times 10^{-3} \mathrm{~s}^{-1}\right)$. Typically, aspect ratios for CNTs are claimed to be larger than 100. However, as CNTs frequently occur in a wavy form, the effective aspect ratios (i.e. those, which allow for an efficient stress transfer) are actually much smaller - thus, three aspect ratios were investigated: 25 (2D and 3D), 50 (2D and 3D) and 100 (2D case only).

As expected, incorporation of CNTs (and their increasing aspect ratio) leads to an increase of Young's modulus $E$ of the nanocomposite of around $4 \%(5.5 \%, 8 \%)$ for 2D RVEs of aspect ratio of $25(50,100)$ and $7.5 \%(9.5 \%)$ for 3D RVEs of aspect ratio of 25 (50) (see Fig.9a). 2D models predicted a stiffer response (in the linear elastic regime) both for pure epoxy and its nanocomposite, when compared to 3D models (see Fig. 8). Therefore, it is concluded that the cause of the stiffer 2D response is the well-known effect of the constraint on the out-of-plane strain caused by the plane strain assumption. The normalised Young's modulus in the 3D case is slightly larger than in the 2D case, probably caused by the better stress transfer due to larger CNT surface area in 3D and the smaller average CNT distance due to the 3D effect.

Values of the parameter $\sigma_{\mathrm{Y}}$ (yield peak stress) show a difference between 2D and 3D models, which is qualitatively similar to those obtained for pure epoxy under plane strain (2D) and 3D conditions (see Fig. 8). However, the CNT presence and the increase in CNT aspect ratio had a negligible effect for 2D RVEs, whereas for 3D RVEs a significant increase from around $4.5 \%$ to $7 \%$ in $\sigma_{\mathrm{Y}}$ was predicted for the increase in CNT aspect ratio from 25 to 50 (see Fig. 9b). The local matrix behaviour of the 2D and 3D models was compared in order to determine the reason for this behaviour. In the averaged true stress-applied strain curves of the matrix one can observe a slight decrease of the yield peak stress in 2D models and the increase of the yield peak stress in 3D models. This can be explained by comparing the CNT distributions in $2 \mathrm{D}$ and $3 \mathrm{D}$ models. For the same volume fraction a denser CNT distribution is generated in $3 \mathrm{D}$ with a much smaller average CNT to CNT distance - a 3D scaffold of densely packed CNTs is compared against well separated CNTs in the 2D plane (see Fig. 10). The dense CNT networks in the 3D RVEs limit the expansion in the lateral directions when the 3D RVEs are subjected to uniaxial loading. Within the matrix, this promotes a bigger volume change as well as an increase in mean stress when compared to pure epoxy. This led to bigger averaged stresses in the matrix and the nanocomposite - a similar effect is imposed by the plane-strain assumption. In contrast, for 2D models with a bigger average CNT to CNT distance, the average mean stress within the matrix around the yield peak was slightly smaller than for the pure epoxy. It is noteworthy to mention that the latter result was also present in 3D models, but the increased mean stress is believed to compensate its effect on the nanocomposite yield peak stress.

Subsequently, a decrease of the magnitude of softening modulus (defined by a reduced softening slope) with increasing CNT aspect ratio was found for 3D RVEs (see Fig. 8), while a small increase of the magnitude of softening modulus was predicted for 2D RVEs. Two main effects were identified to contribute to the reduced softening response in 3D. First, the CNT to CNT distance is reduced (as explained earlier), which causes the increase of the averaged mean stress in the matrix. Secondly, the averaged stress in the CNTs increases well beyond the yield peak within the matrix for the 3D RVE, as shown in Fig. 11. In contrast, the averaged stress within the CNTs for 2D RVEs 
resembles closely the average response of epoxy with regimes such as yield, softening and hardening. The large CNT to CNT distance in 2D RVEs limits the inter-particle interaction, and thus the averaged stress response of CNTs is mainly governed by the behaviour of epoxy. It is believed that the increased inter-particle interaction in 3D RVEs (because of the smaller CNT to CNT distances) changes an epoxy-like behaviour for the CNTs and therefore for the nanocomposite.

It was predicted that increasing CNT aspect ratio accelerates the onset of strain hardening, as indicated by the decreased strain softening limit $\varepsilon_{\mathrm{S}}$ (see Fig. 9c). Changing the aspect ratio from 25 to 50 reduced $\varepsilon_{S}$ both for $2 \mathrm{D}$ and $3 \mathrm{D}$ models around $20 \%$ for $3 \mathrm{D}$, and $7 \%$ for $2 \mathrm{D}$ models. This difference between 2D and 3D models is a further consequence of the difference in nanocomposite morphology, as explained above.

\subsubsection{Effect of CNT volume fraction for aligned and random CNT orientations}

The effect of CNT volume fraction on the compressive true stress-true strain curves for aligned CNTs and randomly oriented CNTs is shown in Figs. 12 and 13 using 2D and 3D RVEs. In each case, the predictions are for RVEs with CNT volume fractions of $0.5 \%$ and $1 \%$ and aspect ratio of 50. They were obtained for the quasi-static strain rate of $1 \times 10^{-3} \mathrm{~s}^{-1}$.

In general, the CNT volume fraction has a qualitatively similar effect on the stress-strain parameters, as the CNT aspect ratio. As expected, the Young's modulus increased linearly with increasing CNT volume fraction (see Fig. 13a). The largest enhancement is associated with 3D nanocomposite morphologies of aligned CNTs with an improvement of $\sim 32 \%$ for CNT volume fraction of $1 \%$, while less than half this value was obtained for its $2 \mathrm{D}$ counterpart (i.e. $~ 14 \%$ increase of Young's modulus for CNT volume fraction of 1\%). This is again caused by the improved stress transfer due to the larger surface area in 3D and the smaller average CNT distance due to the 3D effect. Significantly smaller differences for the Young's modulus between 2D and 3D RVEs were found for random CNT orientations.

An interesting result was observed regarding the effect of volume fraction on the nanocomposite yield peak. In particular, the volume fraction does not affect the yield peak parameter $\sigma_{\mathrm{Y}}$ in 2D models (see Fig. 13b) (similarly to the effect of aspect ratio in 2D). However, for 3D models there is a linear increase in $\sigma_{\mathrm{Y}}$ with increased CNT volume fraction. Larger effects were found for 3D nanocomposite morphologies of aligned CNTs (rather than random) with an increase from 15\% to $33 \%$. This matches the magnitude of the effect of CNT volume fraction on the Young's modulus of the nanocomposite. It is noteworthy to mention that in contrast to 3D nanocomposite morphologies of randomly oriented CNTs, this effect does not arise from the increase in the nanocomposite mean stress. Rather the mean stress within the matrix was actually smaller than for the pure epoxy at applied strains corresponding to the yield peak stress. Instead, it is believed that the major contribution here is brought by the larger stress, which was carried by the 3D CNTs, as shown in Fig. 14, in terms of averaged true stress-applied strain curves for the reinforcement. It is expected that a larger surface area of CNTs within the 3D models contributes to that by promoting a much better stress transfer.

Additionally, it must be mentioned that there is a second averaged CNT stress peak followed by the stress drop located in the hardening regime (at around 35-40\% of applied nominal strain) as predicted by 2D RVEs (see Fig. 14). It originates from intensive bending and rotation of 2D CNTs, which reduces the stress transfer.

Then, a significant decrease in the magnitude of softening modulus (case CNT volume fraction of $0.5 \%$ ) and the disappearance of the softening behaviour (case CNT volume fraction of $1 \%$ ) were predicted by 3D RVEs with aligned CNTs. It is believed that this results from the significant increase of the averaged CNT stress in 3D RVEs, as discussed above and shown in Fig. 14.

The softening strain limit $\varepsilon_{S}$ was predicted to decrease with increasing CNT volume fraction (see Fig. 13c).The corresponding true strain values were found to be larger for 3D RVEs than for 2D RVEs, and also larger for aligned CNTs than for randomly oriented CNTs: (a) for CNT volume 
fraction of $0.5 \%$, a reduction of $20-25 \%$ in $\varepsilon_{S}$ was obtained for 2D and 3D approaches of randomly oriented CNTs, (b) decrease of 40\% (2D) and 55\% (3D) was predicted for aligned CNTs.

\subsubsection{Effect of strain rate for aligned and random CNT orientations}

The effect of strain rate for aligned CNTs and randomly oriented CNTs is shown in Figs. 15 and 16 for strain rates of $1 \times 10^{-3}$ (quasi-static strain rate) and $1 \times 10^{3} \mathrm{~s}^{-1}$ (impact rate of strain). Both figures show predictions of RVEs containing CNTs of aspect ratio set to 50 and volume fraction equal to $1 \%$. The stress-strain parameters in Fig. 16 were normalised against corresponding values of pure epoxy subjected to quasi-static strain rate.

For pure epoxy, the increased strain rate shifted the yield peak stress and the corresponding strain to larger values (see Figs. 15 and 16a). A conventional explanation is that the time window of material deformation becomes smaller than the relaxation time of the material. Similar trends were predicted for the nanocomposite using 2D and 3D RVEs with aligned and randomly oriented CNTs. As recognised in the previous section, nanocomposite morphologies of aligned CNTs in the 3D case exhibit a larger increase of $\sigma_{\mathrm{Y}}$ or $\sigma_{\mathrm{p} 1}$ than morphologies of randomly oriented CNTs. However, the magnitude of this enhancement was larger for impact rates of strain $(\sim 55 \mathrm{MPa})$ than for the quasistatic strain rates ( 35MPa) (see Fig. 15).

Considering the softening strain limit parameter $\varepsilon_{\mathrm{S}}$, its normalised value did not show a significant dependency on the strain rate as predicted by 2D RVEs - for pure epoxy it shifted to slightly smaller values of strain with increasing strain rate.

For $3 \mathrm{D}$ approaches, the effect of strain rate on $\varepsilon_{\mathrm{S}}$ is shown only for nanocomposites of randomly oriented CNTs because the softening regime is no longer present after CNT alignment. In that case, the normalised parameter $\varepsilon_{S}$ was significantly reduced from 0.53 to 0.43 when increasing the strain rate from $1 \times 10^{-3}$ to $1 \times 10^{3} \mathrm{~s}^{-1}$ (see Fig. 16b). For the 3D case with aligned CNTs a significantly larger slope of the curve in the post yield regime with increased strain rate was predicted (see Fig. 15). It is believed that the enhanced inter-particle interaction in 3D RVEs and CNT alignment cause an improved matrix to CNT stress transfer in the post-yield regime with the increased strain rate. This is confirmed by the increase of average stresses within CNTs, extracted from RVE models, as shown in Fig. 17.

\subsection{Computational time}

All simulations were performed using a multi-processor supercomputer. 3D RVEs containing 25 randomly oriented CNTs consisted of around 100000 elements and 800000 degrees of freedom. Typical 3D simulations took around 43 hours of wall-clock time with 60 CPUs and a total CPU time reported by ABAQUS of 364 hours (abort at around $21 \%$ of true strain). In contrast, 2D RVEs incorporating 20 randomly oriented CNTs consisted of around 14000 elements and 56000 degrees of freedom. In this case the wall-clock time was 1.5 hours using 24 CPUs and a total CPU time reported by ABAQUS was 7 hours (abort at around $36 \%$ of true strain).

\subsection{Comparison with experiments}

Some of the predicted stress-strain trends were qualitatively compared with the available experimental data from the literature. Experimental results of Dassios et al. [12] were selected, where the authors investigated the large-strain response of aligned CNT mats impregnated with epoxy under quasi-static compressive loading. Similarly to the predictions using 3D RVE based models with aligned CNTs, the authors observed an increase in Young's modulus and yield peak, and a shift of yielding to larger values of strain for the CNT/epoxy systems, compared to pure epoxy. Due to the relatively large CNT volume fraction of $0.27 \%$ and the special arrangement of 
CNTs in the mat (thickness of the mat is equal to the CNT length), their compression test result represents an upper bound for the current 3D models of aligned CNTs.

\section{Conclusion}

RVE-based modelling of aligned and misaligned (with random orientations) morphologies of randomly distributed CNTs in an epoxy matrix was carried out to investigate the compressive nonlinear, rate-dependent, finite strain response of CNT/epoxy nanocomposites. A particular goal of this work was to compare the 2D RVE-based approach against the 3D RVE-approach to study its accuracy in capturing the behaviour of the nanocomposite and their computational efficiency.

Relatively large differences between predictions of the nonlinear compressive response were obtained from the 2D and the 3D modelling approach. In general, the 2D RVE approach suffers from a lack of inter-particle interaction due to a larger average CNT to CNT distance and the smaller surface area available for the stress transfer from the matrix to CNTs, when compared to their 3D counterparts. As a consequence (1) the 2D RVE expanded easier in the lateral direction which resulted in an inaccurate prediction of the average mean stress in the matrix, and (2) the average CNT stress in 2D RVEs was found to be significantly smaller than for 3D RVEs. The larger surface area in 3D RVEs promotes more stress to be transferred into CNTs, which better exploits their mechanical properties. Additionally, in 2D RVEs the averaged stress-applied strain response of CNTs resembled that of the pure matrix. In contrast, a smaller CNT to CNT distance in 3D RVEs increased inter-particle interaction, which resulted in an increase of the averaged stress in the CNT component during the strain softening phase of the matrix. Thus, the smaller CNT to CNT distance in 3D models shields the CNTs from the behaviour of the matrix.

As a result, the 2D RVEs underestimated the effect of the morphological factors (CNT aspect ratio, CNT volume fraction and CNT orientation) on the salient parameters of stress-strain curves. In particular, the yield peak stress was found to be nearly unaffected by the presence of CNTs and various changes of the morphological factors in the 2D case. In contrast, 3D models predicted significant effects of morphological factors on the yield peak stress. Therefore, despite obvious demands on substantial computational resources (approximately a 70-fold increase in computational time for 3D models), it is nevertheless advisable to use 3D RVEs for accurate prediction of the nanocomposite response.

The 3D modelling approach predicted that incorporation of CNTs into the epoxy matrix leads to an enhancement of the nonlinear deformation that results in: (1) the increase of yield stress with the CNT aspect ratio, CNT volume fraction and CNT alignment, (2) the change from softening to hardening with the CNT alignment and (3) the shift of the onset of the hardening regime to smaller stages of strain with the CNT aspect ratio and the CNT volume fraction. The CNT alignment was found to have the most significant enhancement effect, especially at high strain rates, which highlights the reinforcement potential of CNTs for impact-resistant applications.

Some of the predicted stress-strain trends obtained in this work were qualitatively compared with the available experimental data from the literature. Similarly to the predictions using 3D RVE-based models with aligned CNTs, those experimental results show an increase in Young's modulus and yield peak, and a shift of yielding to larger values of strain for the CNT/epoxy systems compared to pure epoxy [12].

However, it has to be mentioned that in reality, morphologies of CNT based nanocomposites show CNT waviness, CNT agglomerations and frequently also an imperfect bonding between CNT and polymer matrix. Future modelling has to account for those aspects in order to overcome the often observed deviation between experiments and modelling. 


\section{Acknowledgment}

The PhD scholarship from the Irish Research Council (IRC) is gratefully acknowledged. Computational facilities and support were provided by the Irish Centre for High-End Computing (ICHEC). The second author would like to acknowledge the financial support from the National Centre for Science (Poland) through the OPUS programme (Grant No. DEC2011/01/B/ST8/06492).

\section{References}

[1] M.M.J. Treacy, T.W. Ebbesen, J.M. Gibson, Nature 381 (1996) 678-680.

[2] C.A. Cooper, R.J. Young, M. Halsall, Compos. Part A-Appl. S. 32 (2001) 401-411.

[3] A. Peigney, C. Laurent, E. Flahaut, R.R. Bacsa, A. Rousset, Carbon 39 (2001) 507-514.

[4] D. Weidt, L. Figiel, M. Buggy, IOP Conf. Ser.: Mater. Sci. Eng.40 (2012) 12028-12033.

[5] F.H. Gojny, M.H.G. Wichmann, U. Köpke, B. Fiedler, K. Schulte, Compos. Sci. Technol. 64 (2004) $2363-2371$.

[6] W. Zhang, R.C. Picu, N. Koratkar, Appl. Phys. Lett. 91 (2007) 193109.

[7] C.P. Buckley, J. Harding, J.P. Hou, C. Ruiz, A. Trojanowski, J. Mech. Phys. Solids 49 (2001) 1517-1538.

[8] L. Ci, J. Suhr, V. Pushparaj, X. Zhang, P.M. Ajayan, Nano Lett. 8 (2008) 2762-2766.

[9] E.T. Thostenson, T.W. Chou, J. Phys. D Appl. Phys. 35 (2002) L77-80.

[10] C.A. Martin, J.K.W. Sandler, A.H. Windle, M.K. Schwarz, W. Bauhofer, K. Schulte, M.S.P. Shaffer, Polymer 46 (2005) 877-886.

[11] T. Kimura, H. Ago, M. Tobita, S. Ohshima, M. Kyotani, M. Yumura, Adv. Mater. 14 (2002) 1380-1383.

[12] K.G. Dassios, S. Musso, C. Galiotis, Compos. Sci. Technol. 72 (2012) 1027-1033.

[13] P. Jindal, S. Pande, P. Sharma, V. Mangla, A. Chaudhury, D. Patel, B.P. Singh, R.B. Mathur, M. Goyal, Compos. Part B-Eng. (2012) 417-422.

[14] W. Al-Lafi, J. Jin, S. Xu, M. Song, Macromol. Mater. Eng. 295 (2010) 519-522.

[15] P. Raju Mantena, A. Al-Ostaz, A.H. Cheng, Compos. Sci. Technol. 69 (2009) 772-779.

[16] S. Namilae, N. Chandra, Compos. Sci. Technol.66 (2006) 2030-2038.

[17] R. Rafiee, R Maleki Moghadam, Comp. Mater. Sci. 63 (2012) 261-268.

[18] C. Li, T.W. Chou, Compos. Sci. Technol. 66 (2006) 2409-2414.

[19] C.P. Buckley, P.J. Dooling, J. Harding, C. Ruiz, J. Mech. Phys. Solids 52 (2004) 2355-2377.

[20] M.M. Shokrieh, R. Rafiee, Comp. Mater. Sci. 50 (2010) 437-446.

[21] Y. Pan, L. Iorga, A.A. Pelegri, Compos. Sci. Technol. 68 (2008) 2792-2798.

[22] R. Makvandi, A. Öchsner, Comp. Mater. Sci. 77 (2013) 189-193.

[23] P. Barai, G.J. Weng, Int. J. Plasticity 27 (2011) 539-559.

[24] P.C. Ma, J.K. Kim, B.Z. Tang, Compos. Sci. Technol. 67 (2007) 2965-2972.

[25] J.B. Bai, A. Allaoui, Compos. Part A-Appl. S. 34 (2003) 689-694.

[26] A. Hernández-Pérez, F. Avilés, A. May-Pat, A. Valadez-González, P. J. Herrera-Franco, P. Bartolo-Pérez, Compos. Sci. Technol. 68 (2008) 1422-1431.

[27] L. Shen, J. Li, Phys. Rev. B 69 (2004) 045414.

[28] F.H. Gojny, M.H. Wichmann, B. Fiedler, K. Schulte, Compos. Sci. Technol. 65 (2005) 2300-2313.

[29] K.W. Putz, M.J. Palmeri, R.B. Cohn, R. Andrews, L.C. Brinson, Macromolecules 41 (2008) 6752-6756.

[30] H. Wan, F. Delale, L. Shen. Mech. Res. Commun. 32 (2005) 481-489.

[31] A. Hernández-Pérez, F. Avilés, Comp. Mater. Sci. 47 (2010) 926-933.

[32] C. Li, T.W. Chou, J. Nanosci. Nanotechno. 3 (2003) 423-430.

[33] S. Yang, , S. Yu, J. Ryu, J.M. Cho, W. Kyoung, D.S. Han, M. Cho, Int. J. Plasticity 41 (2013) 124-146.

[34] G.D. Ruxton, Behav. Ecol. 17 (2006) 688-690.

[35] J.W. Hutchinson, Z. Suo, Adv. Appl. Mech. 29 (1992) 63-191. 